\title{
Route Reservation in Ad Hoc Wireless Networks
}

\author{
Sooksan Panichpapiboon, Gianluigi Ferrari, Nawaporn Wisitpongphan, and Ozan K. Tonguz
}

\begin{abstract}
This paper investigates whether and when route reservation-based (RB) communication can yield better delay performance than non-reservation-based (NRB) communication in ad hoc wireless networks. In addition to posing this fundamental question, the requirements (in terms of route discovery, medium access control (MAC) protocol, and pipelining, etc.) for making RB switching superior to NRB switching are also identified. A novel analytical framework is developed and the network performance under both RB and NRB schemes is quantified. It is shown that if the aforementioned requirements are met, then RB schemes can indeed yield better delay performance than NRB schemes. This advantage, however, comes at the expense of lower throughput and goodput compared to NRB schemes.
\end{abstract}

Index Terms—Ad hoc wireless networks, resource reservation, performance analysis, bit error rate, goodput, throughput, delay.

\section{INTRODUCTION}

$\mathrm{T}$ HE two principal switching techniques used in wired networks are circuit switching and packet switching [1]. One of the main differences between them is the way resources are shared. Circuit switching provides exclusive access to the resources by means of reservation. In packet switching, on the other hand, resources are shared ondemand, without prior reservation. While it is obvious that packet switching is suitable for a wired data network such as the Internet, it is not clear whether this is true in the case of ad hoc wireless networks. To the best of our knowledge, a direct study and comparison between these two switching schemes for wireless ad hoc and sensor networks has not been reported in the literature so far. In this paper, we investigate the performance of two switching paradigms: reservation-based (RB) and non-reservation-based (NRB) switching. The concepts of reservation and non-reservation are analogous to those of circuit switching and packet switching in wired networks, respectively. However, there are some important differences, which can be summarized as follows:

- In an NRB scheme, an intermediate node can simultaneously serve as relay for more than one source. Hence, the resources (in terms of relaying nodes) are shared in an on-demand fashion. This is typical for most of the routing protocols for wireless ad hoc networks proposed in the literature [2].

- S. Panichpapiboon is with the Department of Engineering Education, King Mongkut's Institute of Technology Ladkrabang, 3 Chalongkrung Road, Ladkrabang, Bangkok 10520, Thailand. E-mail:kpsooksa@kmitl.ac.th.

- N. Wisitpongphan and O.K. Tonguz are with the Electrical and Computer Engineering Department, Carnegie Mellon University, 5000 Forbes Ave., Pittsburgh, PA 15213-3890. E-mail: nawaporn@andrew.cmu.edu, tonguz@ece.cmu.edu.

- G. Ferrari is with the University of Parma, Dipartimento di Ingegneria dell'Informazione, Palazzina 2, Sede Scientifica, Parco Area delle Scienze, 181/A, I-43100 Parma, Italy. E-mail: gianluigi.ferrari@unipr.it.

Manuscript received 14 Jan. 2005; revised 9 Sept. 2005; accepted 27 Apr. 2006; published online 15 Nov. 2006.

For information on obtaining reprints of this article, please send e-mail to: tmc@computer.org, and reference IEEECS Log Number TMC-0009-0105.
- In an RB scheme, a source first reserves a multihop route to its destination, i.e., it reserves intermediate nodes before the actual transmission begins. The reserved intermediate nodes are required to relay only the message generated by the specific source. This gives the source an exclusive access to the path to the destination. This particular route reservation approach for ad hoc wireless networks was first introduced in [3].

In addition to posing the interesting question of whether and when RB switching makes sense in wireless ad hoc networks, in this paper, we develop novel analytical models (queuing models) for analyzing the network performance (in terms of throughput, delay, goodput, and maximum tolerable speed) under the RB and NRB switching schemes. Although some simplifying assumptions are made to keep the analysis tractable, the results presented in this paper still provide significant insights and may stimulate further research in this area.

One of the important contributions of this work is to identify under which conditions (in terms of route discovery, MAC protocol, pipelining, etc.) the delay performance of the RB scheme can be superior to the NRB scheme. While the conventional wisdom in current wireless ad hoc networking research favors NRB switching, in this paper, we show, for the first time, when and under which conditions RB switching might be preferable. Our results show that, even under these somewhat strict and futuristic conditions, while RB switching provides a better delay performance, NRB switching can generally achieve higher network goodput and throughput. It is important to understand that if these conditions are not satisfied, then NRB switching will probably be preferable.

The rest of this paper is organized as follows: In Section 2, we briefly discuss the related work in this area. In Section 3, we describe ad hoc wireless network communication models and assumptions used in this paper. We describe the basic principles of operation of RB and NRB switching schemes in Section 4. Performance of the two switching schemes is analyzed in detail in Section 5. Results and their 
implications are presented in Section 6. Finally, concluding remarks are given in Section 7.

\section{Related Work}

A number of routing protocols for ad hoc wireless networks have been proposed over the past few years. Most of these protocols can be categorized as variants of the NRB routing protocol, where packets are relayed on a route with best effort. Examples include [4], [5], [6], and more references can be found in [2]. A number of studies related to the evaluation of NRB switching schemes have also been reported. In [7], [8], [9], performance of a few routing protocols for ad hoc wireless networks, in terms of throughput, end-to-end delay, and amount of overhead, are investigated using computer simulations. In [10], an analytical model for evaluating the performance, in terms of capacity and throughput, of static ad hoc wireless networks without a delay constraint is proposed. The achievable network throughput for a given delay constraint is then studied in [11] and [12]; however, queuing delay at each node is not taken into consideration. In [13], the authors consider NRB switching in ad hoc wireless networks and derive delay bounds for a 2-hop relay case and a multihop relay case with packet flooding.

Many reservation-based routing protocols are also proposed in the literature. These routing protocols are designed to guarantee quality of service (QoS) such as bandwidth and delay. In [14], a ticket-based probing algorithm is used for searching routes which satisfy bandwidth and delay constraints. In [15], a time division multiple access (TDMA)-based QoS routing algorithm is considered. An IP-based QoS framework for mobile ad hoc networks is presented in [16]. Variants of the ReSerVation Protocol (RSVP) [17], a well-known resource reservation protocol used in the Internet, for mobile wireless networks are proposed in [18], [19]. However, to the best of our knowledge, none of these resource reservation protocols has considered the reservation of intermediate nodes on a multihop route as presented in this paper. In addition, while a few analytical models exist for NRB switched ad hoc wireless networks, similar models have not been reported for RB schemes. In this paper, we propose novel and tractable analytical models for RB and NRB schemes. The performance analysis of RB schemes is first considered in [20] where the effects of interference and retransmission are not taken into account. In this paper, we provide a more rigorous interference analysis and also include a retransmission model.

\section{Network Models and Assumptions}

\subsection{Network Topology}

Throughout the paper, we consider a scenario where $N$ nodes are distributed over a surface with finite area $A$. The node spatial density is defined as the number of nodes per unit area and denoted as $\rho_{\mathrm{s}} \triangleq N / A$ (dimension: $\left[\mathrm{m}^{-2}\right]$ ). To avoid edge effects, we assume the network surface to be the surface of a torus [21]-however, the analytical technique presented in this paper can be applied to other types of surfaces as well. The torus assumption allows us to

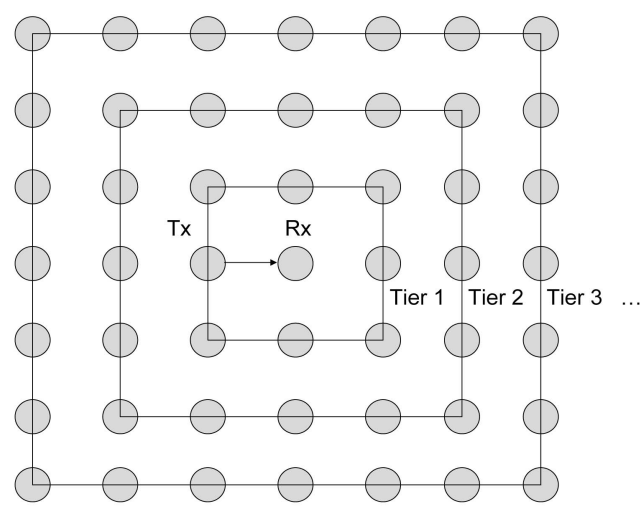

Fig. 1. Tier structure of a grid network.

treat any node in the network the same, whether it is at the edge or at the center of the network. In a real scenario, the performance predicted by our analysis may not be extremely precise for nodes on the edge of the network surface. In this case, a more precise performance evaluation may be obtained via simulations.

In this paper, for getting fundamental insights into this important problem, we consider networks with a square grid topology, where each node has four nearest neighbors. ${ }^{1}$ An example of such network topology is shown in Fig. 1. Due to the structure of the square grid topology, the distance to the nearest neighbor, denoted by $r_{\text {link }}$, is fixed, and a route corresponds to a sequence of hops with equal length. The distance $r_{\text {link }}$ can be computed as follows: Note that constructing a square lattice of $N$ nodes over a surface of a torus with area $A$ is equivalent to fitting $N$ small square tiles of area $r_{\text {link }}^{2}$ into a large square of area $A$. Hence, it follows that $N r_{\text {link }}^{2}=A$. Finally, the distance between two nearest neighbors can be written as [3]

$$
r_{\text {link }}=\sqrt{\frac{A}{N}}=\frac{1}{\sqrt{\rho_{\mathrm{s}}}} .
$$

\subsection{Typical Routes}

In a peer-to-peer ad hoc wireless network, where source/ destination pairs are randomly selected, the number of hops in each route is likely to be different. In this paper, we consider a route with an average number of hops as representative for average network performance evaluation. In other words, we implicitly assume that routes with an average number of hops are typical. We now estimate the average number of hops in a multihop route in a networking scenario with grid topology.

Due to the spatial invariance on a torus, we can assume without any loss of generality that a source node is at the center of the network (see Fig. 1). If a destination node is selected at random, the minimum number of hops to reach the destination can range from 1 to $2 i_{\max }$, where $i_{\max }$ is the maximum tier order. In other words, it takes 1 hop to reach a destination which is a neighbor of a source node in Tier 1 , and it takes $2 i_{\max }$ hops to reach the farthest node from the

1. The analysis can also be extended for the case of a network with random topology by following the approach outlined in [21]. 
center in Tier $i_{\max }$. The average number of hops can be obtained by counting the number of hops on a route from the source to each destination node and finding the average value. Assuming that each destination is equally likely, the average number of hops on a route can be written as

$$
\bar{n}_{\mathrm{h}}=\frac{1}{N-1}\left[4 \sum_{i=1}^{i_{\max }} i+4 \sum_{i=1}^{i_{\max }} 2 i+8 \sum_{i=1}^{i_{\max }} \sum_{j=1}^{i-1}(i+j)\right] .
$$

The first summation term in (2) corresponds to the number of hops to reach any of the four nodes in alignment with the source at the center of the network in all possible tiers; the second summation corresponds to the number of hops to reach nodes on the four corners of each tier; finally, the third term (double summation) corresponds to the number of hops to reach the other nodes in each tier. With straightforward algebra, (2) can be simplified to

$$
\bar{n}_{\mathrm{h}}=\frac{2}{N-1}\left(2 i_{\max }^{3}+3 i_{\max }^{2}+i_{\max }\right)
$$

Since there are $8 i$ nodes in tier $i$, it can be shown that if the number of nodes is sufficiently large, $i_{\max } \simeq \sqrt{N} / 2$. Therefore, for sufficiently large $N$, the average number of hops given by (3) can be approximated as

$$
\bar{n}_{\mathrm{h}} \simeq \frac{\sqrt{N}}{2} .
$$

\subsection{Bit Error Rate at the End of a Multihop Route}

In this section, we derive an expression for bit error rate (BER) at the end of a multihop route, which is essential for the performance analysis presented in the next section. As indicated in the previous section, we assume that a communication route is given by a sequence of links between nearest neighbors, i.e., with equal length. The BER at the end of a link between two neighboring nodes, denoted by $\mathrm{BER}_{\text {link, }}$, depends on the signal-to-noise ratio (SNR) at the receiving node. Generally, the SNR is a function of the transmit power, the distance between transmitter and receiver, pathloss, thermal noise power, and interference power. Assuming that the interfering signals are independent of each other, the SNR at the receiving node of a link can be written as [3], [22]

$$
\mathrm{SNR}_{\text {link }}=\frac{\left[G_{\mathrm{t}} G_{\mathrm{r}} c^{2} /\left(4 \pi f_{\mathrm{c}}\right)^{2}\right] P_{\mathrm{t}}}{r_{\text {link }}^{\gamma} \frac{R_{b}}{B}\left(P_{\text {therm }}+P_{\text {int }}\right)}=\frac{\alpha P_{\mathrm{t}}}{r_{\text {link }}^{\gamma} \frac{R_{b}}{B}\left(P_{\text {therm }}+P_{\text {int }}\right)},
$$

where $G_{\mathrm{t}}$ and $G_{\mathrm{r}}$ are transmitter and receiver antenna gains, $\gamma$ is the pathloss exponent, $f_{\mathrm{c}}$ is the carrier frequency, $P_{\mathrm{t}}$ is the transmit power, $P_{\text {therm }}$ is the additive white Gaussian thermal noise power, $P_{\text {int }}$ is the interference power, $R_{\mathrm{b}}$ is the data rate (dimension: $[\mathrm{b} / \mathrm{s}]$ ), and $B$ is the bandwidth (dimension: [Hz]). The ratio $R_{\mathrm{b}} / B$ corresponds to the spectral efficiency of the used modulation format [22]. The thermal noise power $P_{\text {therm }}$ at the receiver can generally be written as $F k T_{0} B$, where $F$ is the noise figure, $k=$ $1.38 \times 10^{-23} \mathrm{~J} / \mathrm{K}$ is the Boltzman's constant, and $T_{0}=300 \mathrm{~K}$ is the room temperature [23].
The theoretical worst-case interference power in a network with grid topology, corresponding to a scenario where all nodes transmit simultaneously, can be expressed as [24]

$$
P_{\text {int }}=\frac{\alpha P_{\mathrm{t}}}{r_{\text {link }}^{\gamma}} \sum_{i=1}^{i_{\max }}\left[\frac{4}{i^{\gamma}}+\frac{4}{(\sqrt{2} i)^{\gamma}}+\sum_{j=1}^{i-1} \frac{8}{\left(\sqrt{i^{2}+j^{2}}\right)^{\gamma}}-1\right],
$$

where $i$ is the tier order. For simplicity, in this paper, we consider binary phase shift keying (BPSK) signalinghowever, the proposed approach can be straightforwardly extended to any modulation format [22]. This worst-case interference corresponds to having no MAC protocol at all. More discussion on MAC protocols is given in Section 6.6. Since the total interfering noise is the sum of many random signal components, by the Central Limit Theorem (CLT), the total interfering noise is approximately Gaussian [25]. In this case, the link BER can be written as

$$
\mathrm{BER}_{\text {link }}=Q\left(\sqrt{2 \mathrm{SNR}_{\text {link }}}\right),
$$

where $Q(x) \triangleq \frac{1}{\sqrt{2 \pi}} \int_{x}^{\infty} e^{-u^{2} / 2} \mathrm{~d} u$.

Assuming that uncorrected errors accumulate in successive links (a conservative assumption which is accurate at large link SNR values), the BER at the end of an $\bar{n}_{\mathrm{h}}$-hop route can be written as ${ }^{2}$

$$
\mathrm{BER}_{\text {route }} \simeq 1-\left(1-\mathrm{BER}_{\text {link }}\right)^{\bar{n}_{\mathrm{h}}} .
$$

Given that a message is divided into packets of fixed length $l_{\mathrm{p}}$ (dimension: [b/pck]), in the case of independent bit transmission (as is the case for uncoded BPSK signaling), the packet error rate (PER), denoted as $\mathrm{PER}_{\text {link }}$, is related to the link BER as follows:

$$
\mathrm{PER}_{\text {link }}=1-\left(1-\mathrm{BER}_{\text {link }}\right)^{l_{\mathrm{p}}} .
$$

\subsection{Retransmission Model}

For network communications to be reliable, retransmission of packets in error is needed. In this paper, we consider a simple retransmission scheme where a packet in error will be retransmitted up to a maximum number of times, $k_{\max }$. If a packet is still received erroneously after $k_{\max }$ retransmissions, then we assume that the receiver will have to take that packet in its current status.

The maximum number of retransmissions $k_{\max }$ is clearly an important parameter. In particular, in this paper, it reflects the QoS in terms of link PER. If the maximum number of retransmissions is too small, then the PER may not satisfy the desired QoS. The desired QoS will depend on the type of applications. For example, for the transmission of very sensitive data, one may impose a QoS such that every packet must be "error-free." However, for some applications, such as image transmission, every packet may not need to be perfect-some errors in a packet might alter the colors of the image, but the image may still be recognizable. We now derive an expression for $k_{\max }$.

Let $\mathrm{PER}_{\text {link }}^{\max }$ be the maximum tolerable PER required by an application. The objective is to guarantee that after $k_{\max }$ retransmissions, the link PER is lower than $\mathrm{PER}_{\text {link }}^{\max }$.

2. Readers are referred to [3], [22] for additional details regarding the physical layer analysis. 
Assuming that different packet retransmissions are independent of each other, the value $k_{\max }$ is the minimum possible value such that a packet is transmitted successfully with fewer than $k_{\max }$ retransmissions. In other words, $k_{\max }$ is the smallest integer for which the following inequality is satisfied:

$$
\sum_{j=0}^{k_{\max }}\left(\mathrm{PER}_{\text {link }}\right)^{j}\left(1-\mathrm{PER}_{\text {link }}\right) \geq 1-\mathrm{PER}_{\text {link }}^{\max } .
$$

Solving (10) for the lowest possible integer value of $k_{\max }$, one obtains:

$$
k_{\max }=\left\lceil\frac{\ln \left(\mathrm{PER}_{\mathrm{link}}^{\max }\right)}{\ln \left(\mathrm{PER}_{\mathrm{link}}\right)}-1\right\rceil,
$$

where $\lceil\cdot\rceil$ is the ceiling operation. Without considering this operation, i.e., simply considering

$$
k_{\max }=\frac{\ln \left(\mathrm{PER}_{\text {link }}^{\max }\right)}{\ln \left(\mathrm{PER}_{\text {link }}\right)}-1,
$$

it follows that $k_{\max }$ may not necessarily be an integer. However, a noninteger value of $k_{\max }$ can be interpreted as follows: If, for example, $k_{\max }$ is equal to 0.01 , it means that 1 retransmission in every 100 packet transmissions is required to satisfy the imposed QoS. In addition, (11) only makes sense if $\mathrm{PER}_{\text {link }}^{\max }$ is lower than $\mathrm{PER}_{\text {link }}$-that is, if $\mathrm{PER}_{\text {link }}^{\max }$ is higher than $\mathrm{PER}_{\text {link, no retransmission is }}$ required because the QoS is already satisfied.

\subsection{Mobility}

Although the main body of our analysis refers to ad hoc wireless networks with fixed nodes (e.g., sensor networks [26] and wireless mesh networks [27]), it is also important to study how our results could be extended to a scenario with mobile nodes. In particular, we will derive bounds on the maximum node speed which can be tolerated by the considered ad hoc wireless networks for a given packet size, in order for the results derived for a scenario with stationary nodes to still apply.

Intuitively, the transmission of a message along a multihop route can be successfully accomplished without route maintenance [28], [29] if every node in the route does not move "too far" from its neighbors during message transmission. In other words, a route does not break as long as each node does not move out of its neighbors' range. In this section, we quantify the maximum node speed at which a message transmission on a route is not disrupted. We use a simple and conservative mobility scheme. In particular, we assume that a route breaks if a node moves, regardless of the direction, by more than a distance $d_{\max }$, which corresponds to a fraction $\delta<1$ of the hop length $r_{\text {link, }}$, i.e., $d_{\text {max }}=\delta r_{\text {link }}$. Assuming that each node moves at the maximum speed $v_{\max }$, the minimum time it takes for a link to break is

$$
t_{\text {break }}=\frac{d_{\max }}{v_{\max }}=\frac{\delta r_{\text {link }}}{v_{\max }}=\frac{\delta}{v_{\max } \sqrt{\rho_{\mathrm{s}}}} .
$$

A transmission is not disrupted as long as the transmission time of a message is not larger than $t_{\text {break. }}$. Hence, given a desired value for the time $t_{\text {break }}$ to deliver an entire message

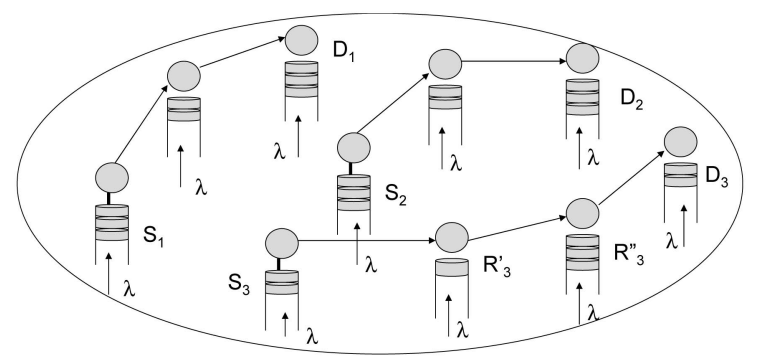

(a)

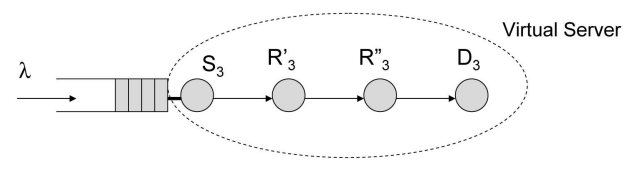

(b)

Fig. 2. Reservation-based ad hoc wireless network model. (a) General scheme: Each node has its own queue, and there are disjoint multihop routes in the network. (b) Equivalent conceptual model for the multihop route between $\mathrm{S}_{3}$ and $\mathrm{D}_{3}$-observe that the queues of the relay nodes $\mathrm{R}_{3}^{\prime}$ and $R_{3}^{\prime \prime}$ and the destination node $\mathrm{D}_{3}$ are suppressed: In other words, the relay nodes and the destination nodes do not involve new queues.

from source to destination, from (12), the maximum tolerable speed $v_{\max }$ for each transmission scheme can then be determined as

$$
v_{\max }=\frac{\delta}{t_{\text {break } \sqrt{\rho_{\mathrm{s}}}}} .
$$

In Section 5, we will evaluate the time it takes for a message to propagate from source to destination in a multihop route, for both RB and NRB schemes. Considering this value as $t_{\text {break, }}$ we will then derive bounds on the maximum tolerable speed (or, for a given maximum speed, the message size that can be transmitted before a route breaks).

\section{The Two Switching Schemes \\ 4.1 Reservation-Based (RB) Switching}

The principle of operation of an RB scheme is fairly simple. Prior to data transmission, a source node reserves a multihop route to the destination through a route discovery phase [28]. We assume that route discovery messages are sent on a separate control channel. Once an intermediate node agrees to relay traffic for a particular source in the network, it cannot initiate a session or relay messages for any other source until the on-going session is over. The source node releases the route after the session ends. We emphasize that this reservation pertains to node processing but not to the shared common radio channel. In other words, the intermediate nodes dedicate their processing time only to the source which reserved the route; however, reservation of a multihop route does not give any node an exclusive access to the shared radio channel (in terms of frequency bands, time slots, or spreading codes). Fig. 2a illustrates an example of reserved routes in a network where an RB scheme is used.

In order to evaluate the performance of an RB switching scheme, we make the following assumptions: 


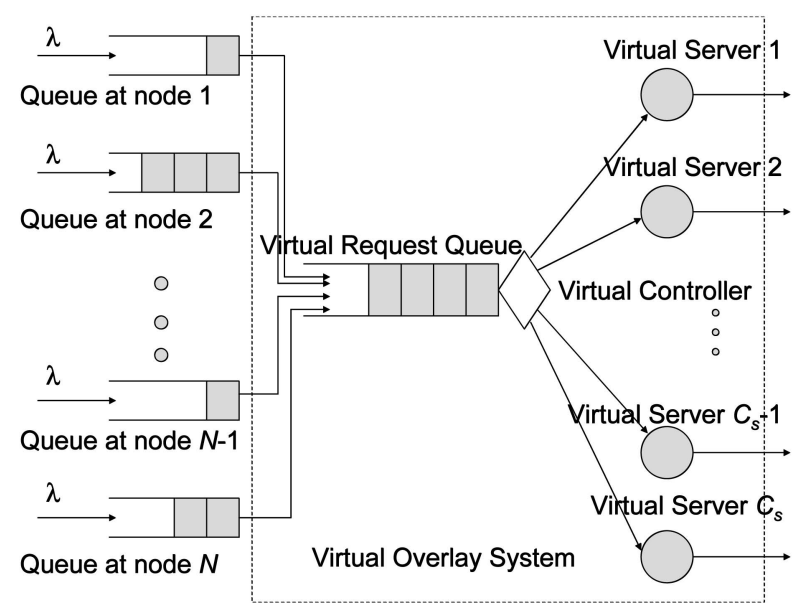

Fig. 3. Conceptual queuing model for a reservation-based wireless network: Real queues at each node are connected to an overall virtual request queue. Each virtual server corresponds to a possible multihop route.

- Each node in the network generates messages according to a Poisson process with average arrival rate $\lambda_{\mathrm{m}}$ (dimension: $[\mathrm{msg} / \mathrm{s}]$ ). While a node is acting as a relay, it still generates its own messages, which are buffered for future transmission.

- The message length $L_{\mathrm{m}}$ is exponentially distributed ${ }^{3}$ with average value $\bar{L}_{\mathrm{m}}$ (dimension: $[\mathrm{b} / \mathrm{msg}]$ ). Considering a fixed transmission data rate $R_{\mathrm{b}}$, the message duration is therefore exponentially distributed with a mean value equal to $\bar{L}_{\mathrm{m}} / R_{\mathrm{b}}$. There are no measurement data on the distribution of message sizes in ad hoc wireless networks. However, the exponential message size in an ad hoc network, for example, could be the duration of a voice communication session, as the duration of a typical voice call has an exponential distribution [30].

- Since intermediate nodes on a multihop route serve only one source node at a time, simultaneously active multihop routes are disjoint. In addition, given that each multihop route has a certain average length, there exists a maximum average number, denoted by $C_{\mathrm{s}}$, of simultaneously active routes (an expression for $C_{\mathrm{s}}$ will be provided in Section 5.1.1).

- If the number of nodes wishing to activate a multihop route is larger than $C_{\mathrm{s}}$, then some nodes have to wait before they can activate the route. The amount of time that a node has to wait before it can activate a route will be referred to as "access delay."

- The route activation process can be described by a conceptual "virtual request queue" which regulates requests from all sources (see Fig. $2 \mathrm{~b}$ and Fig. 3). In this sense, one can imagine that the first message of the queue at each source node is immediately

3. Note that message lengths, in bits, should be characterized by a discrete probability distribution. However, for analytical convenience, in this paper, we describe them with a continuous (exponential) probability distribution. A noninteger value should realistically correspond to its closest integer value. forwarded to the virtual request queue. As will be shown later, the virtual request queue models the waiting time that a source experiences after discovering a route but before being able to activate it. Each possibly active multihop route corresponds, in this conceptual model, to a virtual server which takes care of the messages in the virtual request queue. The number of servers corresponds to the maximum average number $C_{\mathrm{s}}$ of disjoint multihop routes in the network.

- The time spent by a message in the virtual request queue corresponds to the time necessary for intermediate nodes to become available. Therefore, a message in the virtual request queue might not be served in the order in which it arrives. However, according to Little's theorem, the average delay in the system will be the same regardless of the specific queuing discipline employed [31].

- The total delay between generation and complete transmission of a message, at each source node, is obtained by adding three terms: 1) the time spent in the node's own queue (denoted by $W_{\mathrm{o}}^{\mathrm{RB}}$ ), 2) the time spent in the virtual request queue (denoted by $W_{\mathrm{v}}^{\mathrm{RB}}$ ), and 3) the time spent in the server (denoted by $T_{\mathrm{s}}^{\mathrm{RB}}$ ). In particular, the queue at each node can be modeled as an $M / G / 1$ queue with service time $\tau_{\mathrm{RB}}=W_{\mathrm{v}}^{\mathrm{RB}}+T_{\mathrm{s}}^{\mathrm{RB}}$.

- The combination of the virtual request queue and the $C_{\mathrm{s}}$ virtual servers will be denoted as "virtual overlay system." In particular, there are $N$ flows of information at its input, coming from the $N$ nodes. Invoking Kleinrock's independence approximation [1], the total arrival process at the input of the request queue can be modeled as Poisson with rate $N \lambda_{\mathrm{m}}$. Hence, it follows that the virtual overlay system shown in Fig. 3 can be modeled as an $M / M / C_{\mathrm{s}} / \infty / N$ system [32].

\subsection{Non-Reservation-Based (NRB) Switching}

In the case of NRB switching, there is no reservation of a route prior to data transmission. As opposed to an RB scheme, in an NRB network communication scenario, multihop routes can overlap. In particular, a node can serve as a relay node for more than one route. In other words, when a node receives a message from another node (i.e., it acts as a relay), it places that message in its own queue (intermingled with its own generated messages). The messages in the queue are transmitted sequentially (i.e., the priority given to relay and new locally generated messages is the same). An example of routes in a network with an NRB scheme is shown in Fig. 4. As in the case of RB switching, we assume that the message generation process is Poisson and that the message length is exponentially distributed with average value $\bar{L}_{\mathrm{m}}$.

Unlike the case with RB switching (where the relay nodes give absolute priority to the relayed messages, stopping to serve their own messages), each multihop route is a tandem of queues and the whole network can also be viewed as a tandem of queues. As a result, Burke's theorem 


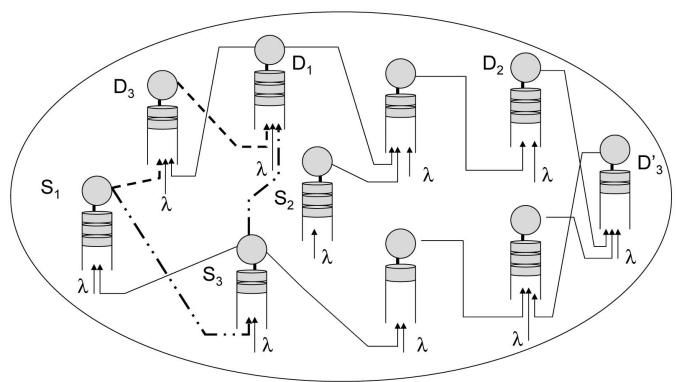

Fig. 4. Non-reservation-based ad hoc wireless network model: Each node has its own queue and the multihop routes are not necessarily disjoint. In particular, two possible multihop routes between $\mathrm{S}_{1}$ and $\mathrm{D}_{1}$ are shown (dashed and dashed-dotted links). Observe that the same source can transmit successive messages to different destination (for example, source $S_{3}$ might be transmitting to destinations $\mathrm{D}_{3}$ and $\mathrm{D}_{3}^{\prime}$ ).

can be applied and each individual node can be modeled as an $M / M / 1$ queue [1]. The conceptual model of an NRB network is shown in Fig. 5.

\section{Analysis of the Two Switching Techniques}

Analytical models for evaluating the performance, in terms of delay, goodput, and throughput, of RB and NRB schemes are presented in the following two sections.

\subsection{Reservation-Based Switching}

\subsubsection{Maximum Number of Disjoint Routes}

One of the key factors that affect the performance of an RB scheme is the maximum number of disjoint routes in the network. This corresponds to the number of virtual servers $C_{\mathrm{s}}$ in the $M / M / C_{\mathrm{s}} / \infty / N$ queuing model described in Section 4.1. Given that each route has an average number of hops $\bar{n}_{\mathrm{h}}$, the maximum number of disjoint routes, corresponding to a scenario where each node belongs to a particular route (i.e., as a source, a relay node, or a destination), can simply be written as

$$
C_{\mathrm{s}} \simeq \frac{N}{\bar{n}_{\mathrm{h}}+1} .
$$

\subsubsection{Delay}

With the assumptions specified in Section 4.1, each node is modeled as an $M / G / 1$ queue. The average delay that each message experiences is equal to the sum of the mean waiting time in the source queue, denoted as $\mathrm{E}\left[W_{\mathrm{o}}^{\mathrm{RB}}\right]$, and the mean service time $\mathrm{E}\left[\tau_{\mathrm{RB}}\right]$, where, as previously defined, $\tau_{\mathrm{RB}}=W_{\mathrm{v}}^{\mathrm{RB}}+T_{\mathrm{s}}^{\mathrm{RB}}$. The mean waiting time in an $M / G / 1$ queue can be computed using the PollaczeckKhinchin formula [1]:

$$
\mathrm{E}\left[W_{\mathrm{o}}^{R B}\right]=\frac{\lambda_{\mathrm{m}} \mathrm{E}\left[\tau_{\mathrm{RB}}^{2}\right]}{2\left(1-\lambda_{\mathrm{m}} \mathrm{E}\left[\tau_{\mathrm{RB}}\right]\right)} .
$$

It is clear from (15) that one needs to compute the first and second moments of the service time $\tau_{\mathrm{RB}}$, which can be derived from the statistics of the total time spent in the $M / M / C_{\mathrm{s}} / \infty / N$ virtual overlay system. The probability density function (pdf) of the time spent in the $M / M / C_{\mathrm{s}} / \infty / N$ system is [32]

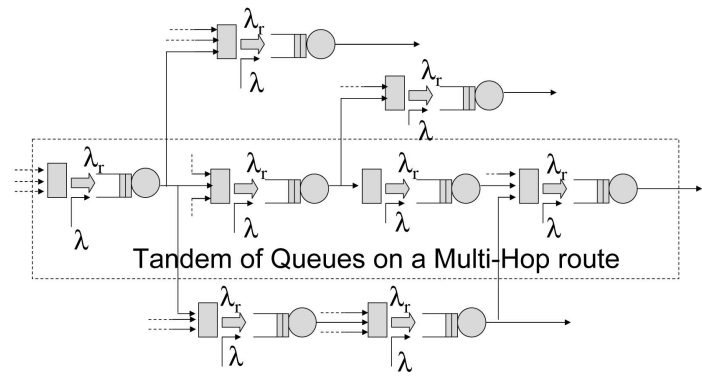

Fig. 5. Conceptual queuing model for a non-reservation-based wireless network: The queues at the nodes of a multihop route constitute a tandem of queues.

$$
\begin{aligned}
f_{\tau_{\mathrm{RB}}}(x)= & \mu e^{-\mu x} \sum_{n=0}^{C_{\mathrm{s}}-1} a_{n}+\sum_{n=C_{\mathrm{s}}}^{N-1} a_{n}\left[\mu e^{-\mu x}\left(\frac{C_{\mathrm{S}}}{C_{\mathrm{s}}-1}\right)^{n-C_{\mathrm{s}}+1}\right. \\
& \left.-\mu\left(\frac{C_{\mathrm{s}}}{C_{\mathrm{s}}-1}\right)^{n-C_{\mathrm{s}}+1} \sum_{r=0}^{n-C_{\mathrm{s}}} e^{-\mu x} \frac{\left[\mu\left(C_{\mathrm{s}}-1\right) x\right]^{r}}{r !}\right],
\end{aligned}
$$

where $a_{n}$ is the probability that a new arrival finds $n$ "customers" in the virtual overlay system (i.e., $n$ nodes are transmitting or waiting to start transmitting an already generated message), and $\mu$ is the average service rate.

The probability distribution $\left\{a_{n}\right\}$ of the number of customers that a new arrival sees in an $M / M / C_{\mathrm{s}} / \infty / N$ system involves the computation of large factorials (e.g., $N$ !), which leads to numerical problems. To analyze a largescale ad hoc network, we exploit the fact that when the number of sources is large, the steady-state probability distribution of an $M / M / C_{\mathrm{s}} / \infty / N$ system follows that of an $M / M / C_{\mathrm{s}}$ system [32]. The first and second moments of the time that each message spends in the system are given by

$$
\begin{gathered}
\mathrm{E}\left[\tau_{\mathrm{RB}}\right]=\frac{1}{\mu}+\frac{\Gamma\left(C_{\mathrm{s}}, \frac{\lambda_{\mathrm{m}}}{\mu}\right)}{\mu C_{\mathrm{s}}-\lambda_{\mathrm{m}}}, \\
\mathrm{E}\left[\tau_{\mathrm{RB}}^{2}\right]=\frac{2}{\mu^{2}}+\frac{2 \Gamma\left(C_{\mathrm{s}}, \frac{\lambda_{\mathrm{m}}}{\mu}\right)}{\left[\mu C_{\mathrm{s}}-\lambda_{\mathrm{m}}\right]^{2}},
\end{gathered}
$$

where

$$
\begin{aligned}
\Gamma\left(C_{\mathrm{s}}, \frac{\lambda_{\mathrm{m}}}{\mu}\right) \triangleq & {\left[\sum_{n=0}^{C_{\mathrm{s}}-1} \frac{\left(\frac{\lambda_{\mathrm{m}}}{\mu}\right)^{n}}{n !}+\frac{\left(\frac{\lambda_{\mathrm{m}}}{\mu}\right)^{C_{\mathrm{s}}}}{C_{\mathrm{s}} !\left(1-\frac{\lambda_{\mathrm{m}}}{\mu C_{\mathrm{s}}}\right)}\right]^{-1} } \\
& \times \frac{\left(\frac{\lambda_{\mathrm{m}}}{\mu}\right)^{C_{\mathrm{s}}}}{C_{\mathrm{s}} !\left(1-\frac{\lambda_{\mathrm{m}}}{\mu C_{\mathrm{s}}}\right)} .
\end{aligned}
$$

Since the route is reserved, it is possible to transmit a message from source to destination using a pipelining method [33]. Assuming that the whole message of length $L_{\mathrm{m}}$ bits is divided into packets of fixed length $l_{\mathrm{p}}$ bits, the total number of packets per message is $L_{\mathrm{m}} / l_{\mathrm{p}}$. Suppose there are $\bar{n}_{\mathrm{h}}$ links on a route from source to destination. The total time to transmit a message with a pipelining method can be computed as 


$$
T_{\mathrm{s}}^{\mathrm{RB}}=\frac{L_{\mathrm{m}}}{R_{\mathrm{b}}}+\left(\bar{n}_{\mathrm{h}}-1\right) \frac{l_{\mathrm{p}}}{R_{\mathrm{b}}} .
$$

Consequently, the total transmission time $T_{\mathrm{s}}^{\mathrm{RB}}$, as given in (20), is not exponentially distributed. This violates the exponential service time assumption. However, if $L_{\mathrm{m}} \gg \bar{n}_{\mathrm{h}} l_{\mathrm{p}}$, then the second term on the right hand side of (20) is negligible, and the exponential service time assumption still holds.

In the case of retransmission, the message transmission time can be generalized as

$$
T_{\mathrm{s}}^{\mathrm{RB}}=\frac{L_{\mathrm{m}}}{R_{\mathrm{b}}}(1+K)+\left(\bar{n}_{\mathrm{h}}-1\right) \frac{l_{\mathrm{p}}}{R_{\mathrm{b}}}(1+K),
$$

where $K$ is the number of retransmissions per link. Since $K$ is a discrete random variable, the transmission time becomes a function of two random variables $\left(L_{\mathrm{m}}\right.$ and $\left.K\right)$. The pdf of the total delay in this case can be written as

$$
f_{T_{\mathrm{s}}^{\mathrm{RB}}}(t)=\sum_{j=0}^{\infty}\left[\frac{\mu e^{-\frac{\mu t}{1+j}}}{1+j}\right]\left[\left(\mathrm{PER}_{\text {link }}\right)^{j}\left(1-\mathrm{PER}_{\text {link }}\right)\right] u(t),
$$

where $u(t)$ is the unit step function, defined as

$$
u(t) \triangleq \begin{cases}1 & t \geq 0 \\ 0 & t<0 .\end{cases}
$$

In particular, (22) can be derived directly from the total probability theorem; the expression in the first square bracket is the conditional pdf of the transmission time given that the number of retransmission is $j$, and the expression in the second square bracket is the probability mass function of the random variable $K$.

The pdf given in (22) is not exponential, and it may not have a closed-form expression. Fortunately, given that the number of retransmissions is $j$, the conditional pdf of the transmission time has the following exponential structure:

$$
f_{T_{\mathrm{s}}^{\mathrm{RB}} / j}(t / j)=\frac{\mu}{1+j} e^{-\frac{\mu t}{(1+j)}} u(t) .
$$

Consequently, even in the case of retransmissions, one can still analyze the delay performance of the network communication system using the same queuing model, but with a modified service time. From (24), it can easily be observed that the new mean service time is $\frac{1+j}{\mu}$. To take advantage of this, the number of retransmissions $j$ must be specified. To be conservative, the number of per-link packet retransmissions which will be used in the following is the maximum number of retransmissions $k_{\max }$ introduced in Section 3.4. In this case, the service time is exponential with mean service time $\frac{\bar{L}_{\mathrm{m}}\left(k_{\max }+1\right)}{R_{\mathrm{b}}}$.

\subsubsection{Goodput}

The goodput is the total amount of bits received correctly per unit time at their respective destinations. Route goodput pertains to the amount of data transported correctly over time on a single multihop route with an average number of hops. Network goodput, on the other hand, is the aggregate amount of goodput due to all routes. It measures how much error-free data can collectively be transferred in a network over time. Route goodput, denoted as $\beta$, and network goodput, denoted as $\eta$, can be written as follows, respectively:

$$
\begin{gathered}
\beta \triangleq \lambda_{\mathrm{m}} \bar{L}_{\mathrm{m}}\left(1-\mathrm{BER}_{\text {route }}\right), \\
\eta \triangleq \beta \mathrm{E}\left[N_{\mathrm{ar}}\right],
\end{gathered}
$$

where $\mathrm{E}\left[N_{\mathrm{ar}}\right]$ is the expected number of active routes. The expected number of active routes is equal to the expected number of "busy servers" in an $M / M / C_{\mathrm{s}}$ queuing model, which, in this case, is equal to $\frac{N \lambda_{\mathrm{m}}}{R_{\mathrm{b}} / \bar{L}_{\mathrm{m}}}$.

\subsubsection{Throughput}

In order to capture the effects of packet retransmission according to the imposed QoS on link PER (i.e., PER $\mathrm{R}_{\mathrm{th}}$ ), we use throughput instead of goodput. Throughput measures the rate at which a packet is received at its destination. In the case of no retransmission, the rate at which the packets are delivered to the destination is equal to the packet generation rate $\lambda_{\mathrm{p}}$ (dimension: [pck/s]). In the case of retransmissions, the throughput decreases because multiple copies of the same packet are transmitted. The worst-case throughput, where each packet requires $k_{\max }$ retransmissions, can be expressed as

$$
\nu \triangleq \frac{\lambda_{\mathrm{p}}}{\bar{n}_{\mathrm{h}} k_{\max }+1} .
$$

Note that when $k_{\max }$ is equal to zero, (27) corresponds to the case of no retransmission. The network throughput, denoted as $\zeta$, can be computed by adding the throughput of all the active routes, obtaining

$$
\zeta \triangleq \nu \mathrm{E}\left[N_{\mathrm{ar}}\right]
$$

\subsection{Non-Reservation-Based Switching}

\subsubsection{Average Number of Routes per Node}

In an NRB scheme, a node can relay traffic generated by multiple sources. However, the stability condition requires that the total incoming traffic rate is lower than the service rate. Consequently, it is important to know how much traffic a node carries for other sources. With the uniform traffic assumption (i.e., every node generates approximately the same amount of traffic), in order to compute the average traffic relayed by a node, it is sufficient to compute the average number of routes passing through it. In this section, we derive an expression for this number.

Considering a grid topology as shown in Fig. 6, we want to find the average number of routes passing through a generic node, say, node $\mathrm{V}$. We start by finding the probability that node $\mathrm{V}$ belongs to a route of a particular source/destination pair. Let $\mathrm{S}_{i, j}$ be a source node at position $(i, j)$ relative to the position of a node $\mathrm{V}$, where $i$ is the number of hops in the horizontal direction and $j$ is the number of hops in the vertical direction. Due to the spatial invariance on a torus, one can assume, without loss of generality, that the source is at the origin and node $\mathrm{V}$ is at position $(i, j)$ relative to the source, as shown in Fig. 6. We assume that a source can select any node in the network, at 


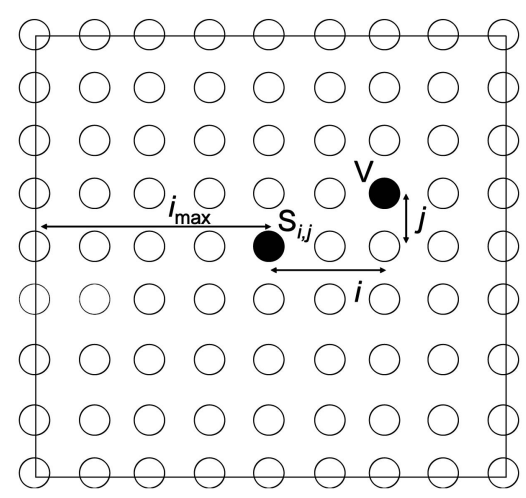

Fig. 6. Node $\mathrm{V}$ represents a possible relay node for source node $\mathrm{S}_{i, j}$.

position $(x, y)$ relative to the source, as its destination. In addition, it is assumed that the shortest route between source and destination is used. In the case that there is more than one shortest route, a source selects one of these routes randomly. For conciseness, let us denote the probability that node $\mathrm{V}$ is on a route of $\mathrm{S}_{i, j}$ as $\mathrm{P}[\mathrm{V}(i, j)]$, and let us denote the conditional probability that $\mathrm{V}$ is on a route of $\mathrm{S}_{i, j}$, given that $\mathrm{S}_{i, j}$ selects a destination at $(x, y)$, as $\mathrm{P}[\mathrm{V}(i, j) / \mathrm{D}(x, y)]$. With these assumptions, one obtains

$$
\mathrm{P}[\mathrm{V}(i, j) / \mathrm{D}(x, y)]= \begin{cases}0 & x<i \text { or } y<j \\ \frac{n_{\mathrm{p}}}{n_{\mathrm{t}}} & \text { otherwise }\end{cases}
$$

where $n_{\mathrm{p}}$ is the number of all possible shortest paths to the destination passing through node $\mathrm{V}$, and $n_{\mathrm{t}}$ is the number of all possible shortest paths to the destination. In the case where $x<i$ or $y<j, \mathrm{P}[\mathrm{V}(i, j) / \mathrm{D}(x, y)]$ is equal to zero because $\mathrm{V}$ cannot possibly be on the shortest path from $\mathrm{S}_{i, j}$ to the destination node at $(x, y)$. In the remaining cases, using combinatorics, it can be shown that

$$
\begin{gathered}
n_{\mathrm{p}}={ }^{i+j} \mathcal{C}_{i}{ }^{x+y-i-j} \mathcal{C}_{x-i}, \\
n_{\mathrm{t}}={ }^{x+y} \mathcal{C}_{x},
\end{gathered}
$$

where ${ }^{n} \mathcal{C}_{r} \triangleq \frac{n !}{(n-r) ! r !}$. The next step is to find the probability that $\mathrm{V}$ is on a route of $\mathrm{S}_{i, j}$ without conditioning on the destination. Using the law of total probability [25], one obtains:

$$
\mathrm{P}[\mathrm{V}(i, j)]=\sum_{\forall(x, y)} \mathrm{P}[\mathrm{V}(i, j) / \mathrm{D}(x, y)] \mathrm{P}[\mathrm{D}(x, y)],
$$

where $\mathrm{P}[\mathrm{D}(x, y)]$ is the probability that a node at position $(x, y)$ relative to $\mathrm{S}_{i, j}$ is selected as the destination. Assuming that a source randomly chooses a destination, $\mathrm{P}[\mathrm{D}(x, y)]$ is simply equal to $1 /(N-1)$. Combining the expressions given in (29)-(32), one gets

$$
\mathrm{P}[\mathrm{V}(i, j)]=\frac{(i+j) !}{i ! j !(N-1)} \sum_{x=i}^{i_{\max }} \sum_{y=j}^{i_{\max }} \frac{(x+y-i-j) ! x ! y !}{(x+y) !(y-j) !(x-i) !}
$$

By neglecting edge effects (according to the torus assumption), we can assume that node $\mathrm{V}$ is at the center of the network. The average number of routes passing through $\mathrm{V}$ can now be computed as

$$
n_{\mathrm{r}}=\sum_{\forall(i, j)} \mathrm{P}[\mathrm{V}(i, j)] .
$$

Next, we assume that each node in the network generates approximately the same amount of traffic according to a Poisson process with average rate $\lambda_{\mathrm{m}}$ (dimension: [msg/s]). Then, the average rate of the traffic entering a node is given as

$$
\lambda_{\text {total }}=\lambda_{\mathrm{m}}+\sum_{i=1}^{n_{\mathrm{r}}} \lambda_{\mathrm{m}}=\left(n_{\mathrm{r}}+1\right) \lambda_{\mathrm{m}}
$$

\subsubsection{Delay}

Since each source does not have a dedicated route, a message transmitted in a route will experience, in addition to the transmission delay, a queuing delay at each node it traverses. According to the assumptions in Section 4.2 (in particular, the applicability of Burke's theorem to an NRB switching network [1]), the average delay that a packet experiences at each node it traverses corresponds to that of an $M / M / 1$ queue and is given by

$$
\mathrm{E}\left[T^{\mathrm{NRB}}\right]=\frac{1}{\frac{R_{\mathrm{b}}}{\bar{L}_{\mathrm{m}}}-\lambda_{\text {total }}} .
$$

The total average delay for a message from the source node to the destination node of a multihop route is obtained as the sum of the average delays experienced at each intermediate node. In other words, $\mathrm{E}\left[T_{\text {total }}^{\mathrm{NRB}}\right]=\bar{n}_{\mathrm{h}} \mathrm{E}\left[T^{\mathrm{NRB}}\right]$.

As discussed earlier, given that the number of retransmissions is $k_{\max }$, the service time for transmitting a packet is still exponentially distributed. If a message (exponentially distributed) of size $L_{\mathrm{m}}$ bits is divided into packets of (fixed) length $l_{\mathrm{p}} \mathrm{b} / \mathrm{pck}$, the time it takes to transmit a single packet is $\left(1+k_{\max }\right) l_{\mathrm{p}} / R_{\mathrm{b}}$. Since there are $L_{\mathrm{m}} / l_{\mathrm{p}}$ packets in a message, the time at which the last packet will reach the next hop is $\left(1+k_{\max }\right) L_{\mathrm{m}} / R_{\mathrm{b}}$. Hence, in the case with retransmission, the expected per-link message transmission time in (36) can be generalized as

$$
\mathrm{E}\left[T^{\mathrm{NRB}}\right]=\frac{1}{\frac{R_{\mathrm{b}}}{\bar{L}_{\mathrm{m}}\left(1+k_{\max }\right)}-\lambda_{\text {total }}} .
$$

\subsubsection{Goodput}

Route goodput and network goodput of an NRB switching network can be computed in the same manner as that of an RB scheme. Note that, in the calculation of route goodput, the amount of traffic should correspond to that generated only by source nodes (i.e., excluding the relay traffic). Since there are $N$ sources, the network goodput of an NRB network can then be written as

$$
\eta=\lambda_{\mathrm{m}} \bar{L}_{\mathrm{m}}\left(1-\mathrm{BER}_{\text {route }}\right) N .
$$

\subsubsection{Throughput}

The throughput of an NRB scheme can be computed in the same manner as in the case of an RB scheme. 
TABLE 1

Major Network Parameters Used in the Considered Scenarios

\begin{tabular}{|l|c|l|c|}
\hline Parameters & Values & Parameters & Values \\
\hline \hline Transmit power $\left(P_{\mathrm{t}}\right)$ & $1 \mathrm{~mW}$ & Pathloss exponent $(\gamma)$ & 3 \\
\hline Data rate $\left(R_{\mathrm{b}}\right)$ & $1 \mathrm{Mb} / \mathrm{s}$ & Carrier frequency $\left(f_{\mathrm{c}}\right)$ & $2.4 \mathrm{GHz}$ \\
\hline Average message length $(m)$ & $10^{6} \mathrm{bits}$ & Room temperature $\left(T_{0}\right)$ & $300 \mathrm{~K}$ \\
\hline Packet length $\left(l_{\mathrm{p}}\right)$ & $10^{3} \mathrm{bits}$ & Noise figure $(F)$ & $6 \mathrm{~dB}$ \\
\hline Area of the network $(A)$ & $10^{6} \mathrm{~m}^{2}$ & & \\
\hline
\end{tabular}

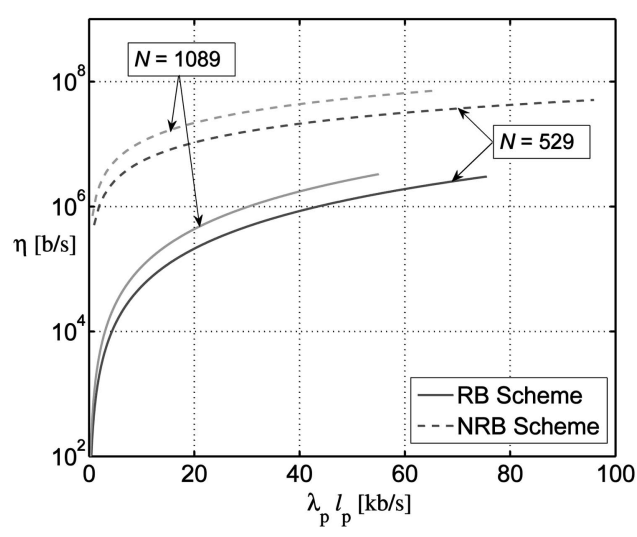

(a)

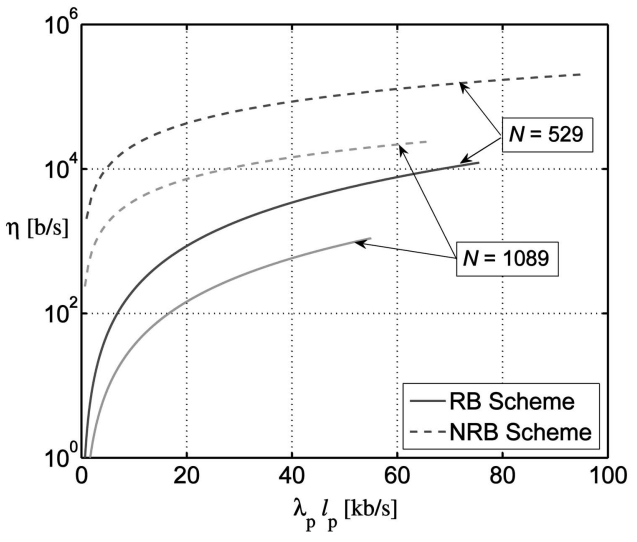

(b)

Fig. 7. Goodput of the two switching schemes in (a) the ideal case and (b) the worst case. The network goodput of the RB scheme and the NRB scheme obtained from the analysis are shown with the solid lines and with the dashed lines, respectively.

\section{Results AND Discussion}

In this section, numerical results are presented and their implications are discussed. In all the considered scenarios, the main network parameter values used in the analysis are shown in Table 1, unless stated otherwise.

\subsection{Switching Scheme and Traffic Load}

In this section, we discuss the trade-off, in terms of goodput and delay, between the two described switching schemes. In Fig. 7, the network goodput of the considered switching schemes, computed via (26) and (38), is shown as a function of traffic load in 1) the ideal case, where interference is neglected, and 2) the theoretical worst case, where all nodes transmit simultaneously, respectively. The worst-case interference power is computed via (6). It is expected that the performance of a realistic ad hoc wireless network will be somewhere between these two cases-in particular, case 1) is overly pessimistic, and performance in a real scenario is expected to be definitely better. Two different values of the number of nodes in the network are considered, as indicated in the figure.

Note that, when the area of the network is fixed, increasing the number of nodes corresponds to a node spatial density increase. Generally, it is observed that the network goodput of an NRB switching scheme is higher than that of the corresponding RB scheme at every value of traffic load and in each scenario (either ideal or worst case). This is due to the fact that an NRB scheme can support a higher number of routes (i.e., the disjoint routes constraint is not imposed in the NRB switching scheme). In addition, observe that the performance difference between the two switching schemes increases, although not significantly, in each scenario (either ideal or worst case), as the network becomes denser.

In Fig. 8 , the average packet delay, denoted as $t_{\mathrm{p}}$, is shown as a function of the average packet generation rate $\lambda_{\mathrm{p}}$ for different values of the number of nodes $N$. The average delay per packet is defined as the arithmetic average between 1) the time the first packet in the message arrives at the destination and 2) the time that the last packet in the message arrives at the destination. The message delay for the RB switching scheme is computed via (15), (17), (18),

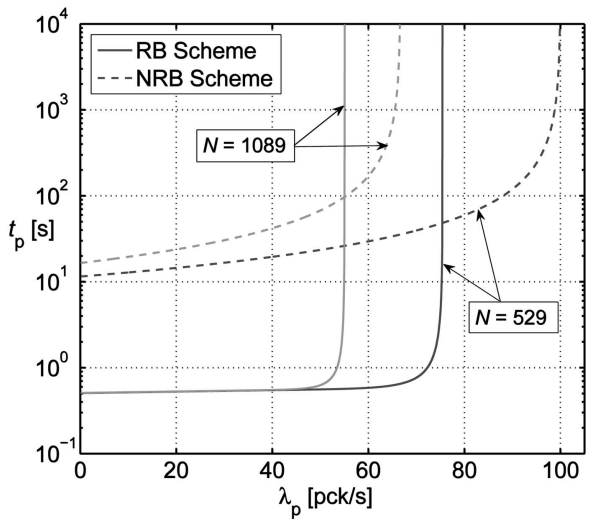

Fig. 8. Delay comparison of the two switching schemes. The average route delay of the RB and the NRB schemes obtained from the analysis are shown with the solid lines and the dashed lines, respectively. 


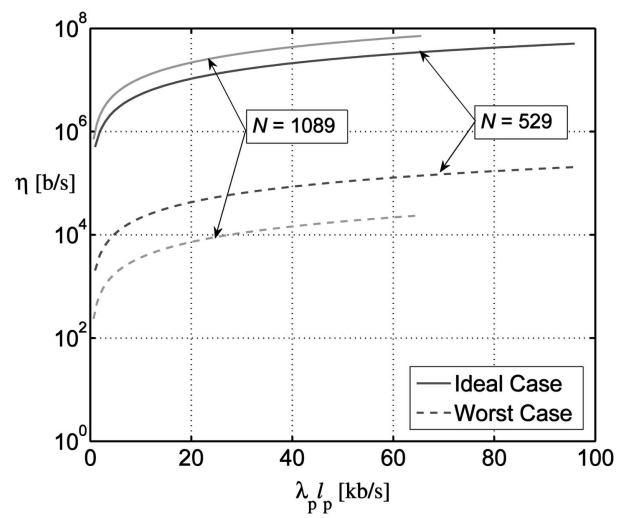

Fig. 9. Goodput of the NRB scheme compared at different node densities. The network goodput for the ideal case and the worst case obtained from the analysis are shown with the solid lines and the dashed lines, respectively.

and (20), whereas the message delay at each node on the route for the NRB switching scheme is computed via (36). As expected, the delay increases as the traffic load increases. The results in Fig. 8 show that an RB scheme performs better than an NRB scheme up to a critical load, which makes the delay of the RB scheme go to infinity very rapidly. Below this critical load, the delay of an RB scheme is very insensitive to the traffic load. The overall behavior of an RB scheme can therefore be characterized as bimodal: either almost constant, with respect to the traffic load, or infinite. Fig. 8 shows that the delay of an RB scheme is lower than that of an NRB scheme by more than one order of magnitude for every considered node spatial density. Nonetheless, the maximum traffic load that an RB scheme can support is lower than that of an NRB scheme. This is due to the disjoint routes constraint. It is important to observe, however, that the difference between the maximum traffic loads that the two schemes can support decreases as the node spatial density increases. In other words, an RB switching scheme becomes preferable, delaywise, in dense ad hoc wireless networks.

\subsection{Effects of Interference}

In this section, we discuss the impact of the interference on the network performance. Fig. 9 illustrates the network goodput of an NRB scheme as a function of the traffic generation rate given by the product $\lambda_{\mathrm{p}} l_{\mathrm{p}}$ (dimension: $[\mathrm{b} / \mathrm{s}]$ ). Both the ideal-case and the worst-case interference scenarios are considered. The network goodput of the RB and the NRB schemes obtained from the analysis are shown with the solid lines and the dashed lines, respectively.

Generally, from Fig. 9, it can be observed that the network goodput increases as the traffic generation rate increases in both ideal and worst case scenarios. This is due to our simplifying assumption that "none or all" nodes interfere. However, in a real scenario, the interference would certainly depend on the traffic generation rate. In fact, the interference is expected to increase as the traffic load increases. There would be a clear trade-off between supporting high traffic load and keeping the interference low. Our current model does not take this aspect into account yet, but this issue is currently under investigation.

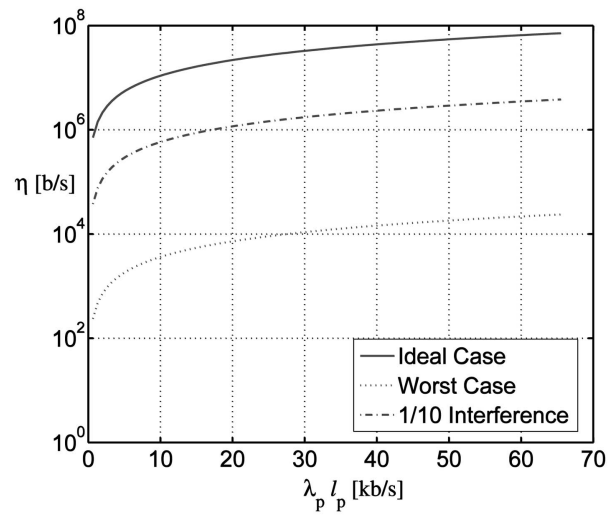

Fig. 10. Goodput of the NRB scheme compared at different levels of interference.

However, the actual performance would still be bounded between those of the ideal and worst cases, as shown here in Fig. 9.

It is observed that the separation between the two bounds (ideal and worst cases) increases as the number of nodes in the network increases. This is reasonable because, keeping the area fixed, the amount of interference is expected to increase as the number of interfering nodes increases (i.e., the network becomes denser). For a large number of nodes (e.g., $N=1,089$ ), the two bounds are separated by almost four orders of magnitude. This clearly indicates that the network, especially if very dense, cannot function well without the use of a MAC protocol effective in canceling or mitigating the interference. The study of such MAC protocols is currently under investigation. The same behavior is observed also in the case of RB schemes.

In order to understand the impact of the interference on the network performance, we also consider cases where the interference power is reduced. Fig. 10 illustrates the network goodput of an NRB scheme computed via (38) as a function of the average bit generation rate, i.e., $\lambda_{\mathrm{p}} l_{\mathrm{p}}$. Three possible cases for the interference level are considered in this figure: 1) no interference (ideal case), 2) worst-case interference, and 3) a scenario where the interference power is one-tenth of the worst case. The number of nodes is fixed at $N=1,089$. Interestingly, the network goodput increases by about two orders of magnitude when the worst-case interference power is reduced to one-tenth. A similar behavior is also observed in the case of RB schemes.

Reduction of the total interference power could be obtained, for example, by dividing the common (shared) communication channel into subchannels (e.g., in frequency, time, or code space). Only nodes using the same subchannel would interfere with one another. As a result, the total interference power would decrease. In general, a MAC protocol should be designed in such a way that the total interference is reduced and the network goodput becomes close to the ideal performance bound. As an example, a MAC protocol which can guarantee low interference level and is practical for an RB switching scheme could be one which uses code division multiple access (CDMA) and per-route spreading codes [34]. In this case, it is possible to show that the interference power is 


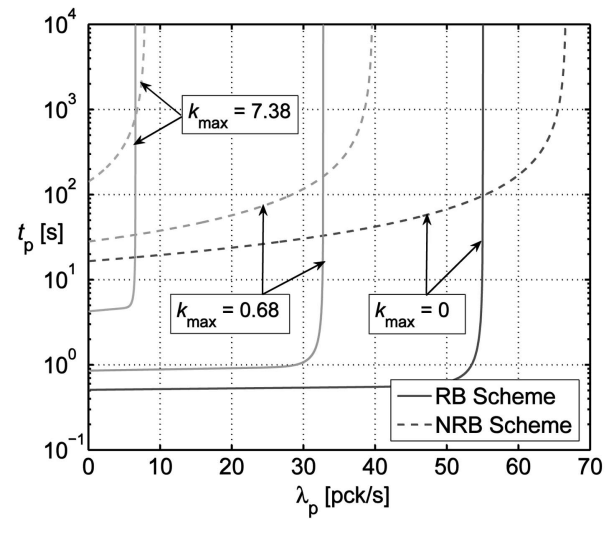

Fig. 11. Delay of the two switching schemes compared at different levels of interference. The average route delay of the RB and the NRB schemes obtained from the analysis are shown with the solid lines and the dashed lines, respectively.

reduced proportionally to the spreading factor of the considered spreading codes.

Fig. 11 illustrates the impact of the interference on the delay performance of the two switching schemes. The message delays for RB and NRB are first computed via (21) and (37), respectively. Then, the average packet delay $t_{\mathrm{p}}$ is computed using the approach described earlier for Fig. 8. Different values of the number of retransmissions $k_{\max }$ for keeping $\mathrm{PER}_{\text {th }}$ above $10^{-2}$ are considered. An increase of $k_{\max }$ can be interpreted as an increase of the interference level. In other words, higher interference requires a higher number of retransmissions per packet. In general, one can conclude that the average packet delay of the two schemes increases as the traffic load increases. In addition, as the level of interference increases, the delay also increases due to a higher number of retransmissions.

The results in Fig. 11 show that the delay of an RB scheme is lower than that of an NRB scheme by more than one order of magnitude for every considered value of $k_{\max }$. Nonetheless, the maximum traffic load that an RB scheme can support is lower than that of an NRB scheme. This is due to the constraint on disjoint routes. It is important to clarify, however, that the difference between the maximum traffic loads that the two schemes can support decreases as the number of retransmissions increases. In other words, an RB switching scheme becomes preferable, delaywise, in an environment where the interference is high.

Fig. 12 illustrates the impact of the interference on the throughput of the two switching schemes. The network throughput of the two schemes is compared for different values of the number of retransmissions $k_{\max }$ that keep $\mathrm{PER}_{\text {th }}$ above $10^{-2}$. It can be observed that the throughput of both schemes decreases as the number of retransmissions increases. However, the throughput of the NRB scheme is larger than that of the RB scheme, due to the higher number of active multihop routes.

\subsection{Effects of the Number of Simultaneously Active Disjoint Routes}

In an RB switching scheme, the number of simultaneously active disjoint routes is an important factor, which significantly affects network performance. Based on our

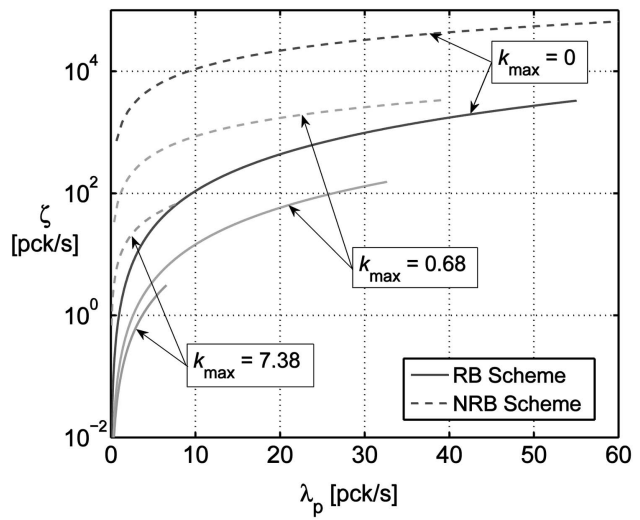

Fig. 12. Throughput of the two switching schemes compared at different levels of interference.

analysis, the maximum number of routes, $C_{\mathrm{s}}$, that can simultaneously be active is, on average, given by (14). In reality, however, the maximum number of disjoint routes that can concurrently be active may not be as large as the average number obtained with our analysis. In this section, we show how the number of active routes affects the performance of the RB switching scheme. In other words, we try to understand what would happen in a realistic scenario where the number of active routes is lower than the theoretical maximum value.

In Fig. 13, the delay of an RB switching scheme is evaluated for three different values of the number of disjoint active routes: 1 ) the maximum average number of routes, $C_{\mathrm{s}}$, estimated from the analysis, 2) half of the maximum number, i.e., $C_{\mathrm{s}} / 2$, and 3 ) one-tenth of the maximum number, i.e., $C_{\mathrm{s}} / 10$. It is observed that, as the maximum number of simultaneously active routes increases, the total amount of traffic which can be supported by the network also increases. In addition, it is interesting to see that the delay behavior of an RB scheme is still insensitive to the amount of traffic load-provided, of course, that the traffic load is sufficiently lower than the critical value.

In Fig. 13, the delay performance of an NRB scheme is also shown for comparison. In general, the delay of an RB

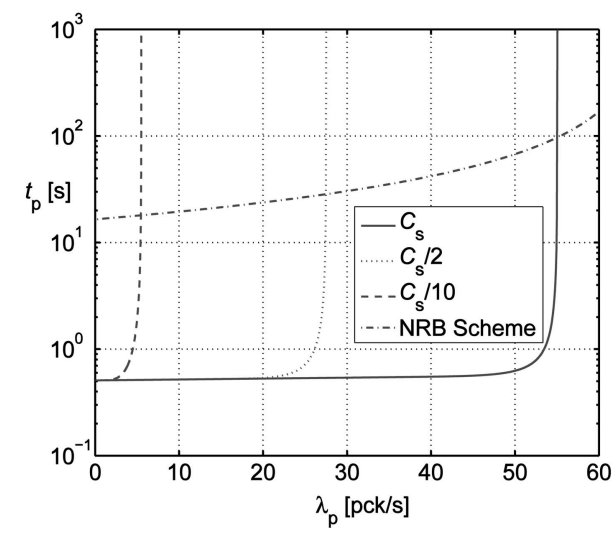

Fig. 13. Delay of a reservation-based scheme (in the ideal case without interference) compared at different values of maximum number of simultaneous active routes. 
TABLE 2

Average Number of Simultaneously Active Routes Obtained from Simulations

\begin{tabular}{|l|c|}
\hline Network Size & Average Number of Active Routes \\
\hline \hline 25 nodes & 4.278 \\
\hline 36 nodes & 5.268 \\
\hline 49 nodes & 6.249 \\
\hline
\end{tabular}

scheme is smaller than that of an NRB scheme. However, an NRB scheme can support much higher traffic load compared to an RB scheme, especially when the maximum number of simultaneously active routes is small. In a real network, the average number of simultaneously active routes is expected to be lower than that of the torus network.

To gain some additional insights, we have also performed simulations to see the average number of simultaneously active routes in the real grid network. In the simulation conducted, we have assigned a random destination for each node in the network. A route from each source node to its destination is established one by one. In the end, we count how many routes can simultaneously be active. This procedure was repeated a sufficiently large number of times, and then we computed the average number of simultaneously active routes. The results are shown in Table 2. It can be observed from Table 2 that the average number of simultaneously active routes in a real network is approximately $\sqrt{N}$, which is about half of the number of active routes in a torus network. More graph-theoretic results on the number of disjoint routes in graphs can be found in [35].

\subsection{Effects of Mobility}

The performance of the two switching schemes, in terms of maximum tolerable speed $v_{\max }$, as defined in Section 3.5, is shown in Fig. 14. Two different values of average message lengths are considered: $\bar{L}_{\mathrm{m}}=1 \mathrm{Mb} / \mathrm{msg}$ and $\bar{L}_{\mathrm{m}}=0.1 \mathrm{Mb} / \mathrm{msg}$, respectively. In this scenario, $\delta$ is equal to 0.1 . It can be observed that, in general, an RB scheme is more robust to node mobility, i.e., it can tolerate a higher maximum node speed. More precisely, from the figure, one can observe that an RB scheme can support communication in a scenario where nodes move at a pedestrian speed (up to $3 \mathrm{~m} / \mathrm{s}$ ) or even at a vehicle speed (up to $25 \mathrm{~m} / \mathrm{s}$ for short message size), whereas an NRB scheme can hardly support any mobility. This behavior can be explained as follows: In an RB scheme, since the route is reserved, it takes less time to transmit a message. Consequently, the total allowed time before a route breaks is larger than that allowed by an NRB scheme, in which an intermediate node has to serve multiple routes. Note that the actual load where the maximum tolerable speed drops to zero corresponds to the load where the delay explodes. In addition, one can observe that the maximum tolerable speed to avoid route breaking increases as the message size decreases.

Alternatively, Fig. 14 can also be interpreted as follows: For a given speed $v_{\max }$ and a traffic load, there is a

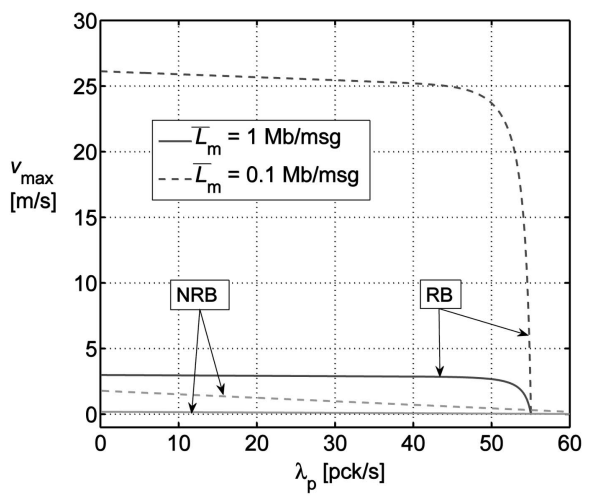

Fig. 14. Effect of mobility.

maximum tolerable average message length at which a message can reach the destination before a communication route breaks. Depending on types of nodes in ad hoc wireless networks (e.g., pedestrians, cars), $v_{\max }$ will be different. As $v_{\max }$ increases, the message length has to decrease correspondingly. One could argue that, in certain applications, it may be impossible to control the speed $v_{\max }$ with which mobile nodes move. The results in Fig. 14 suggest that if $v_{\max }$ and the traffic generation rate are known, then the message length $\bar{L}_{\mathrm{m}}$ should be equal to or less than a fixed value so that the message can reach the destination before the route breaks.

Fig. 14 also suggests that moderate mobility does not significantly affect the goodput and delay performance of an RB scheme. In other words, the goodput and delay performance of an RB scheme presented earlier does not change much if mobile nodes move at moderate speeds (lower than $v_{\max }$ ). On the other hand, the performance of an NRB scheme, in terms of goodput and delay, will significantly degrade if mobile nodes move even slowly, since the maximum tolerable speed is basically zero.

\subsection{Validation of the Queuing Delay Models}

To verify that the conceptual analytical models developed for the RB and NRB switching schemes are correct, we perform independent Monte Carlo simulations for a network employing the two switching schemes. We emphasize that no assumptions about the queuing models (i.e., as in Fig. 3 and in Fig. 5) are made in the simulations.

In the simulations, we assume a $\sqrt{N} \times \sqrt{N}$ square grid network on the surface of a torus. Each node in the network generates messages according to a Poisson process, with mean $\lambda_{\mathrm{m}}[\mathrm{msg} / \mathrm{s}]$. The message length is exponentially distributed. Each message from the source node is routed hop-by-hop to a randomly assigned destination using the $\mathrm{RB}$ and the NRB switching schemes described in Section 4. As in the analysis, we assume that route discovery is done on a separate control channel.

In Fig. 15, the average message delay obtained from the simulations and the average message delay obtained from the analysis for a 25-node network employing RB switching are compared. All the simulations are run on a Microsoft Windows XP machine with a $2.66 \mathrm{GHz}$ Intel Pentium 4 processor. Each point on the simulation curve is the average of 10 simulation trials, where more than 50,000 message 


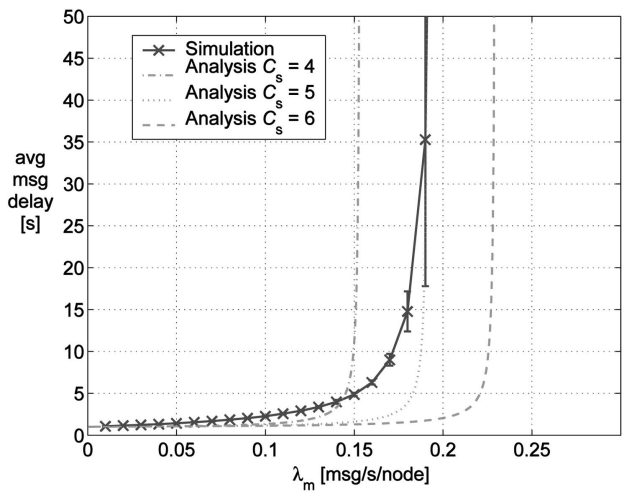

Fig. 15. Delay performance of the RB scheme in a 25-node network. The average message transmission time is 1 second. The simulation results are shown with 95 percent confidence intervals.

arrivals are generated in each simulation trial. The actual time taken to obtain all the points shown on the simulation curve in Fig. 15 is approximately 24.8 hours.

According to our analytical framework, on average, each route has $\sqrt{25} / 2 \approx 3$ hops and, thus, the number of nodes on each route is 4 . The maximum number of simultaneously active routes is $C_{\mathrm{s}}=25 / 4 \approx 6$ routes. The delay predicted by the analytical framework for three different values of $C_{\mathrm{s}}$ are shown in Fig. 15. It can be observed from the figure that the simulation results are close to the delay predicted by the analytical framework with $C_{\mathrm{s}}=5$. However, the delay obtained from the simulation is larger than what is predicted by the analysis. This is because the plot for the analytical model assumes that there are $C_{\mathrm{s}}=5$ routes available at all times. However, in the simulations, there are times when the network cannot support five simultaneously active routes (e.g., some routes could be blocked).

In Fig. 16, the delay performance for the RB scheme and the delay performance for the NRB scheme in a 25-node network are compared. The actual time taken to obtain all the points shown on the RB curve is approximately 24.8 hours, and the actual time taken to obtain all the points on the NRB curve is approximately 28 minutes. Observe that there is a good agreement between the trends predicted by the analytical framework and the Monte Carlo simulation results: In other words, up to a critical traffic load, the delay performance of the RB scheme is superior to that of the NRB scheme.

As a simple sanity check, one can observe the case with extremely low traffic load in Fig. 16. Since the traffic load is very low, the waiting time in the queues is negligible, and the total message delay should therefore be close to the transmission delay. Recall that, in a 25-node network, each route has, on average, 3 hops. In the case with NRB switching, when the arrival rate is near zero, the average message delay is close to 3 seconds. This makes sense because the message transmission time on each link is 1 second, so for a 3-hop route, it takes 3 seconds for a message to reach the destination. In the case with RB switching, when the arrival rate is small, the average message delay is very close to 1 second, which is the message transmission time. This makes sense because even though each route comprises 3 hops, pipelining is used in this case. Hence, the total average message delay should be very close to 1 second.

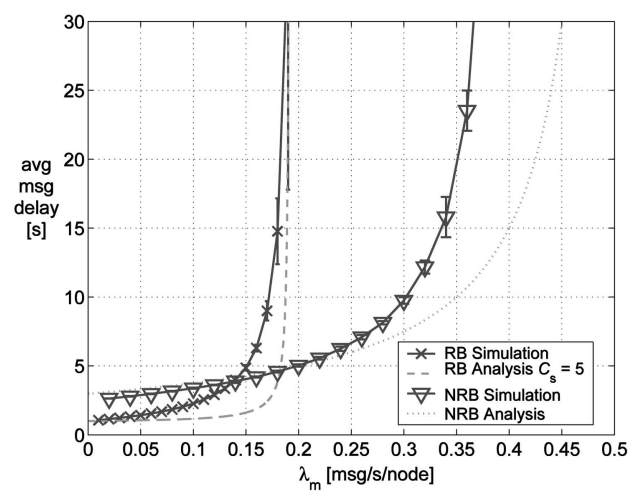

Fig. 16. Delay performance of the RB scheme and the NRB scheme in a 25 -node network. The average message transmission time is 1 second. The simulation results are shown with 95 percent confidence intervals.

\subsection{Implications on Practical Scenarios}

In the analysis presented in this paper, we have made a number of important simplifying assumptions to keep the analysis tractable. In this section, we briefly discuss how the performance of RB and NRB schemes will be affected in a realistic scenario.

- Network topology-We have assumed a square grid topology over the surface of a torus, which allows us to treat any node the same in the analysis. In a real ad hoc network, nodes near the edge of the network are likely to have higher number of hops to the destination than nodes near the center of the network. Thus, the delay for these nodes could be higher than what is predicted by our analysis. In addition, if the network topology is random, the average number of hops in a route could be different from those in a square grid topology. We refer interested readers to [36] for more details on this subject.

- MAC protocol-MAC protocol has not been taken into account in the analytical model presented in this paper. This has implications for the interference and the delay. In terms of interference, we have considered the two extremes: 1) the ideal case where there is no interference and 2) the worst case where all nodes transmit simultaneously. In an RB scheme, the ideal case could be realized if a CDMA scheme with per-route spreading codes is used. This would eliminate the interference among different active routes. However, interference can still occur among nodes within the same route. To reduce the intraroute interference, each node of the same route could transmit on different frequencies. The same frequency can be reused for links in the route that are sufficiently far apart. With this integration of spreading codes and frequencies, the interference could be reduced to a level that is close to the ideal case. Similarly, in the NRB scheme, the ideal case could be realized if each link uses a spreading code that is different from the other links. The same code can also be reused if the links using the same code are sufficiently far apart. Distributed code assignment algorithms for multihop networks, for example, can be found in [37], [38]. The same conclusions also hold if frequency reuse is considered. 
The worst-case interference corresponds to having no MAC protocol at all. Obviously, a real ad hoc wireless network cannot function without a MAC protocol, and any MAC protocol employed should yield a higher goodput. Thus, the worst-case interference could be used as a benchmark for performance comparison [39].

Without any MAC protocol considered, the queuing models in this paper have not taken multiple access delay into account yet. It remains to be seen how delay from a specific MAC protocol employed would affect the performance of RB and NRB schemes. However, for the RB scheme, with the ideal MAC protocol mentioned earlier (i.e., integration of per-route spreading code and use of different frequencies on each hop of a route), it is possible to transmit packets with the pipelining method. This corresponds to the scenario considered in our queuing model and, thus, the total delay can be predicted by the model. Similarly, for the NRB scheme, if the suggested ideal MAC protocol (i.e., different code for each link with possible code reuse) is employed, then the total delay is already captured by our queuing model. If other MAC protocols are used, then one has to take the multiple access delay into account.

- Pipelining-The pipelining packet transmission is assumed in the RB scheme. A MAC protocol that could work under pipelining is suggested in the previous bullet. However, the pipelining assumption implies that a node has to be able to receive and transmit at the same time. This requires a node to have at least two air interfaces. Currently, some wireless communication devices, e.g., cellular phones, already have two air interfaces: one for communicating with a base station in a cellular band and the other for communicating with a Bluetooth device in the unlicensed industrial scientific and medical (ISM) band. Thus, in the near future, nodes in ad hoc wireless networks could also have multiple wireless interfaces, making pipelining possible.

- Interference-In the analysis, we have pessimistically assumed the worst-case scenario, where all nodes transmit simultaneously. In addition, since the total interference noise is the sum of many random signal components, invoking the Central Limit Theorem, we assume that the total interfering noise is Gaussian. However, if the number of interfering nodes is small, this Gaussian assumption could lose its validity and the interference model needs to be reconsidered. Further research is needed to study the impact of these issues on both the RB and NRB schemes [39].

- Route discovery-In our model and the presented analysis, we have neglected this phase of communication, which, in reality, may have a significant impact on the overall delay experienced by a source node. However, the conclusions drawn in this paper regarding the delay performance of $\mathrm{RB}$ and $\mathrm{NRB}$ schemes will not change provided that the delay in discovering a route is similar for both schemes. This happens, for example, if route discovery messages flood the network on a separate control channel in the same way for both switching schemes.

\section{Conclusions}

In this paper, we have posed and investigated an interesting question: namely, if and when reservation-based switching makes sense in contemporary wireless ad hoc networks. While conventional wisdom in current ad hoc wireless networking research favors routing schemes corresponding to the NRB switching, it is shown that if the right requirements (in terms of route discovery, MAC protocol used, pipelining, etc.) are met, then RB switching schemes can provide better delay performance than NRB switching schemes. In return, the throughput and goodput performance of NRB schemes, even under these somewhat stringent requirements, seem to be superior to RB schemes.

Another major contribution of this paper is the novel analytical framework and model (queuing models) developed for analyzing the network performance (in terms of throughput, goodput, delay, and mobility) under the RB and NRB switching schemes. This seems significant as such models, to the best of our knowledge, do not exist (even for NRB schemes) in the open literature.

The results of the analytical framework (also supported by the Monte Carlo simulations conducted) show that RB schemes are appropriate for real-time applications, such as voice and video, whereas NRB schemes are more appropriate for delay-insensitive applications. While RB schemes can provide better delay performance, NRB schemes support higher traffic loads than RB schemes. In addition, it is found that NRB schemes can support a higher number of routes because there is no constraint for the routes to be disjoint. Finally, it is shown that RB schemes are more robust to node mobility than NRB schemes.

We would like to emphasize that the foregoing conclusions are drawn based on the assumptions made in this paper. It is important to understand that if one uses a different MAC protocol (such as 802.11b) and/or one does not use a separate control channel for route discovery, for instance, then the results obtained might be very different from those derived in this paper. In fact, if the requirements outlined in this paper for RB switching are not met, then NRB schemes will probably be preferable.

\section{ACKNOWLEDGMENTS}

The authors are grateful to the three anonymous reviewers of this paper and the associate editor, Professor P.R. Kumar of the University of Illinois at Urbana-Champaign, whose constructive comments and suggestions helped to improve the manuscript substantially. In addition, the authors would like to thank Hsin-Mu Tsai of Carnegie Mellon University (CMU) for his help on the simulations with OPNET. This work was supported in part by an ARO Grant to Cylab of CMU.

\section{References}

[1] D. Bertsekas and R. Gallager, Data Networks. Prentice Hall, 1992.

[2] E.M. Royer and C.-K. Toh, "A Review of Current Routing Protocols for Ad Hoc Mobile Wireless Networks," IEEE Personal Comm. Magazine, vol. 6, no. 2, pp. 46-55, Apr. 1999.

[3] G. Ferrari and O.K. Tonguz, "Performance of Ad Hoc Wireless Networks with Aloha and PR-CSMA MAC Protocols," Proc. IEEE GLOBECOM, pp. 2824-2829, Dec. 2003.

[4] C.E. Perkins and E.M. Royer, "Ad-Hoc On-Demand Distance Vector Routing," Proc. IEEE Workshop Mobile Computing Systems and Applications, pp. 90-100, Feb. 1999. 
[5] D.B. Johnson and D.A. Maltz, "Dynamic Source Routing in Ad Hoc Wireless Networks," Mobile Computing, T. Imielinski and H. Korth, eds., pp. 153-181, 1996.

[6] V. Park and M.S. Corson, "A Highly Adaptive Distributed Routing Algorithm for Mobile Wireless Networks," Proc. IEEE INFOCOM, pp. 1405-1413, Apr. 1997.

[7] S.-J. Lee, M. Gerla, and C.-K. Toh, "A Simulation Study of TableDriven and On-Demand Routing Protocols for Mobile Ad Hoc Networks," IEEE Network, vol. 13, no. 4, pp. 48-54, Jul.-Aug. 1999.

[8] S. Das, R. Castaneda, and J. Yan, "Simulation-Based Performance Evaluation of Routing Protocols for Mobile Ad Hoc Networks," ACM Mobile Networks and Applications, vol. 5, no. 3, pp. 179-189, Sept. 2000.

[9] A. Boukerche, "Performance Evaluation of Routing Protocols for Ad Hoc Wireless Networks," ACM Mobile Networks and Applications, vol. 9, no. 4, pp. 333-342, Aug. 2004.

[10] P. Gupta and P.R. Kumar, "The Capacity of Wireless Networks," IEEE Trans. Information Theory, vol. 46, no. 2, pp. 388-404, Mar. 2000.

[11] N. Bansal and Z. Liu, "Capacity, Delay, and Mobility in Wireless Ad-Hoc Networks," Proc. IEEE INFOCOM, vol. 2, pp. 1553-1563, Apr. 2003.

[12] E. Perevalov and R. Blum, "Delay-Limited Throughput of Ad Hoc Networks," IEEE Trans. Comm., vol. 52, no. 11, pp. 1957-1968, Nov. 2004.

[13] M.J. Neely and E. Modiano, "Capacity and Delay Tradeoffs for Ad-Hoc Mobile Networks," Proc. Int'l Conf. Broadband Networks (BROADNET), pp. 428-438, Oct. 2004.

[14] S. Chen and K. Nahrstedt, "Distributed Quality-of-Service Routing in Ad Hoc Networks," IEEE J. Selected Areas in Comm., vol. 17, no. 8, pp. 1488-1505, Aug. 1999.

[15] C.R. Lin and J. Liu, "QoS Routing in Ad Hoc Wireless Networks," IEEE J. Selected Areas in Comm., vol. 17, no. 8, pp. 1426-1438, Aug. 1999.

[16] S. Lee, G.-S. Ahn, X. Zhang, and A.T. Campbell, "INSIGNIA: An IP-Based Quality of Service Framework for Mobile Ad Hoc Networks," J. Parallel and Distributed Computing, vol. 60, pp. 374406, Apr. 2000.

[17] L. Zhang, S. Deering, D. Estrin, S. Shenker, and D. Zappala, "RSVP: A New Resource Reservation Protocol," IEEE Network, vol. 7, no. 5, pp. 8-18, Sept. 1993.

[18] A. Talukdar, B. Badrinath, and A. Acharya, "MRSVP: A Resource Reservation Protocol for an Integrated Services Network with Mobile Hosts," ACM Wireless Networks, vol. 7, no. 1, pp. 5-19, Jan. 2001.

[19] C.-C. Tseng, G.-C. Lee, and R.-S. Liu, "HMRSVP: A Hierarchical Mobile RSVP Protocol," Proc. IEEE Int'l Conf. Distributed Computing Systems Workshops (ICDCS), pp. 467-472, Apr. 2001.

[20] S. Panichpapiboon, G. Ferrari, N. Wisitpongphan, and O.K. Tonguz, "Route Reservation in Ad Hoc Networks: Is It a Good Idea?" Proc. IEEE Wireless Comm. and Networking Conf. (WCNC), pp. 2045-2050, Mar. 2005.

[21] S. Panichpapiboon, G. Ferrari, and O.K. Tonguz, "Sensor Networks with Random versus Uniform Topology: MAC and Interference Considerations," Proc. IEEE Vehicular Technology Conf. (VTC), pp. 2111-2115, May 2004.

[22] G. Ferrari, B. Baruffini, and O.K. Tonguz, "Spectral EfficiencyConnectivity Tradeoff in Ad Hoc Wireless Networks," Proc. Int'l Symp. Information Theory and Its Applications (ISITA '04), pp. 451456, Oct. 2004

[23] T.S. Rappaport, Wireless Communications Principles and Practice. Prentice Hall, 1996.

[24] S. Panichpapiboon, G. Ferrari, and O.K. Tonguz, "Optimal Transmit Power in Wireless Sensor Networks," IEEE Trans. Mobile Computing, vol. 5, no. 10, pp. 1432-1447, Oct. 2006.

[25] A. Papoulis and S.U. Pillai, Probability, Random Variables and Stochastic Processes, fourth ed. McGraw-Hill, 2002.

[26] I. Akyildiz, W. Su, Y. Sankarasubramaniam, and E. Cayirci, "A Survey on Sensor Networks," IEEE Comm. Magazine, vol. 40, no. 8, pp. 102-114, Aug. 2002.

[27] B. Schrick and M.J. Riezenman, "Wireless Broadband in a Box," IEEE Spectrum, vol. 39, no. 6, pp. 38-43, June 2002.

[28] C.E. Perkins, Ad Hoc Networking. Addison-Wesley, 2001.

[29] S. Park and B. Van Voorst, "Anticipated Route Maintenance (ARM) in Location-Aided Mobile Ad Hoc Networks," Proc. IEEE GLOBECOM, pp. 2809-2813, Nov. 2001.
[30] G. Tunnicliffe, A. Murch, A. Sathyendran, and P. Smith, "Analysis of Traffic Distribution in Cellular Networks," Proc. IEEE Vehicular Technology Conf. (VTC), pp. 1984-1988, May 1998.

[31] L. Kleinrock, Queuing Systems Vol. 1. John Wiley \& Sons, 1975.

[32] J. Medhi, Stochastic Models in Queuing Theory. Elsevier Science Publishers, 2003.

[33] J. Kurose and K. Ross, Computer Networking: A Top-Down Approach, second ed. Addison-Wesley, 2002.

[34] N. Abramson, "Multiple Access in Wireless Digital Networks," Proc. IEEE, vol. 82, pp. 1360-1370, Sept. 1994.

[35] J. Kleinberg and E. Tardos, "Approximations for the Disjoint Paths Problem in High-Diameter Planar Networks," J. Computer and System Sciences, vol. 57, pp. 61-73, 1998.

[36] R. Hofstad, G. Hooghiemstra, and D. Znamenski, Distances in Random Graphs with Finite Mean and Infinite Variance Degrees, Feb. 2003, preprint, http://www.win.tue.nl/ rhofstad/research.html.

[37] L. Hu, "Distributed Code Assignments for CDMA Packet Radio Networks," IEEE/ACM Trans. Networking., vol. 1, no. 6, pp. 668677, Dec. 1993.

[38] J. Garcia-Luna-Aceves and J. Raju, "Distributed Assignment of Codes for Multihop Packet-Radio Networks," Proc. IEEE Military Comm. Conf. (MILCOM), pp. 450-454, Nov. 1997.

[39] O.K. Tonguz and G. Ferrari, Ad Hoc Wireless Networks: A Communication-Theoretic Perspective. Wiley and Sons, 2006.

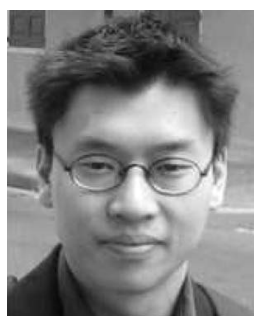

Sooksan Panichpapiboon (S'05) received the $\mathrm{BS}, \mathrm{MS}$, and $\mathrm{PhD}$ degrees in electrical and computer engineering from Carnegie Mellon University (CMU), Pittsburgh, Pennsylvania, in 2000,2002 , and 2006, respectively. $\mathrm{He}$ is currently a faculty member in the Department of Engineering Education at King Mongkut's Institute of Technology, Ladkrabang, Bangkok, Thailand. His research interests include ad hoc wireless networks, sensor networks, medium access control, and performance modeling.

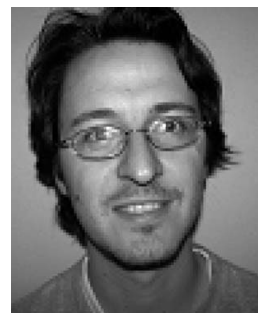

Gianluigi Ferrari (S'97-M'03) received the "Laurea" degree (five year program) (summa cum laude) and the PhD degree in electrical engineering from the University of Parma, Italy, in October 1998 and January 2002, respectively. From July 2000 to December 2001, he was a visiting scholar at the Communication Sciences Institute, University of Southern California, Los Angeles. Since 2002, he has been a research professor in the Department of Information Engineering, University of Parma, where he is now the coordinator of the Wireless Ad-hoc and Sensor Networks (WASN) Laboratory. Between 2002 and 2004, he visited the Electrical and Computer Engineering Department at Carnegie Mellon University, Pittsburgh, Pennsylvania, several times as a research associate. He has published more than 70 papers in leading international conferences and journals. $\mathrm{He}$ is coauthor of the books Detection Algorithms for Wireless Communications, with Applications to Wired and Storage Systems (John Wiley \& Sons, 2004), Teoria della Probabilità e Variabili Aleatorie con Applicazioni (McGraw-Hill, 2005), and Ad Hoc Wireless Networks: A Communication-Theoretic Perspective (John Wiley \& Sons, 2006). He is listed in Marquis "Who's Who in the World," "Who's Who in Science and Engineering," Madison "Who's Who in the World," and in "2000 Outstanding Intellectuals of the 21st Century," International Biographical Centre, United Kingdom. His research interests include digital communication systems design, adaptive signal processing (with particular emphasis on iterative detection techniques for channels with memory), information theory, and ad hoc wireless networking. He was a corecipient of a best student paper award at the 2006 International Workshop on Wireless Ad Hoc Networks (IWWAN '06). He acts as a frequent reviewer for many international journals and conferences. $\mathrm{He}$ also acts as a technical program member for several international conferences (IEEE ICC, IEEE WirelessCom, IEEE IWCMC, IEEE WCNC, Accessnets, IWWAN, IASTED CSA, IASTED WOC, IEEE SECON, IASTER CSNA, SympoTIC). 


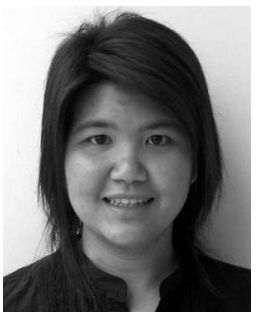

Nawaporn Wisitpongphan received the BS and MS degrees in electrical and computer engineering from Carnegie Mellon University (CMU), Pittsburgh, Pennsylvania, in 2000 and 2002, respectively. Currently, she is working toward the $\mathrm{PhD}$ degree in electrical and computer engineering at CMU. Since 2003, she has been a research assistant at $\mathrm{CMU}$, where she has joined the General Motors collaborative research lab and has been working on designing a routing framework for vehicular ad hoc wireless networks (VANET). Her research interests include traffic modeling, chaos in Internet, and cross-layer network protocol design for wireless networks.

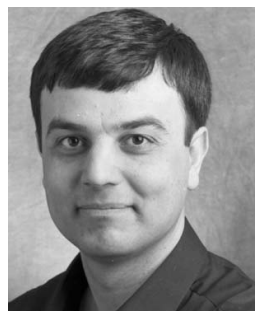

Ozan K. Tonguz received the BSc degree from the University of Essex, England, in 1980, and the MSc and PhD degrees from Rutgers University, New Brunswick, New Jersey, in 1986 and 1990, respectively, all in electrical engineering. From 1988 to 1990 , he was with Bell Communications Research (Bellcore) doing research in optical networks and communication systems. In 1990, he joined the Electrical and Computer Engineering Department at the State University of New York at Buffalo (SUNY/Buffalo) as an assistant professor, where he was granted early tenure and promoted to associate professor in 1995 and to full professor in 1998. He currently serves as a tenured full professor in the Department of Electrical and Computer Engineering at Carnegie Mellon University (CMU), which he joined in August 2000. His current research interests are in wireless networks and communication systems, high-speed networking (Internet), optical networks, satellite communications, bioinformatics, and security. He has published about 200 technical papers in IEEE journals and conference proceedings. He is well-known for his contributions in optical networks (optical transmission-access-networking, especially radio over fiber networks, coherent lightwave transmission systems, and amplified direct-detection lightwave transmission sytems). His recent work on iCAR (the Integrated Cellular and Ad Hoc Relay Systems) is internationally acclaimed as well. $\mathrm{He}$ is the author of the book Ad Hoc Wireless Networks: A Communication-Theoretic Perspective (Wiley and Sons, 2006) and was also the architect of the "High Performance Waveform (HPW)" that was implemented in the Harris RF Communications' AN/PRC-117f UHF band man-pack tactical radio. His industrial experience includes periods with Bell Communications Research, CTI Inc., Harris RF Communications, Aria Wireless Systems, Clearwire Technologies, Nokia Networks, Nokia Research Center, Neuro Kinetics, Asea Brown Boveri (ABB), General Motors (GM), and Intel. He currently serves as a consultant for several companies, law firms, and government agencies in the United States and Europe in the broad area of telecommunications and networking. $\mathrm{He}$ is also a codirector (thrust leader) of the Center for Wireless and Broadband Networking Research at Carnegie Mellon University. More details about his research interests, research group, projects, and publications can be found at http://www.ece.cmu.edu/ tonguz/. In addition to serving on the technical program committees of several IEEE conferences (such as INFOCOM, GLOBECOM, ICC, VTC, WCNC) and symposia in the area of wireless communications and optical networks, Dr. Tonguz currently serves or has served as an associate editor for the IEEE Transactions on Communications, IEEE Communications Magazine, and IEEE Journal of Lightwave Technology. He was a guest editor of the special issue of the IEEE Journal of Lightwave Technology and the IEEE Journal on Selected Areas in Communications on multiwavelength optical networks and technology published in 1996, and a guest editor of the special issue of the Journal of Mobile Multimedia on advanced mobile technologies for health care applications (2006).

$\triangleright$ For more information on this or any other computing topic, please visit our Digital Library at www.computer.org/publications/dlib. 